\title{
Estudos de Caso da aplicação de Métodos Formais da Engenharia de Produção na Engenharia Cartográfica e de Agrimensura
}

Case Studies of Production Engineering's formal methods applications in the Cartography and Surveying Engineering

\author{
Sérgio Orlando Antoun Netto ${ }^{1}$ \\ Jose Carlos Penna de Vasconcellos ${ }^{1}$
}

\begin{abstract}
${ }^{1}$ Departamento de Engenharia Cartográfica, Faculdade de Engenharia, Universidade do Estado de Rio de Janeiro UERJ. Rua São Francisco Xavier 524, 5 andar, Campus Maracanã. CEP 20550-900. Rio de Janeiro, RJ, Brasil. sergio.uerj@gmail.com,jcpvasco@gmail.com
\end{abstract}

Recebido em 20/09/2015 - Aceito em 11/04/2016

Received on 09/20/2015 - Accepted on 04/11/2016

\begin{abstract}
RESUMO - A Engenharia de Produção se dedica ao projeto e gerência de sistemas que envolvem pessoas, materiais, equipamentos e o ambiente. Dentre as subáreas do conhecimento relacionadas à Engenharia de Produção destaca-se a Pesquisa Operacional, que trata da resolução de problemas reais envolvendo situações de tomada de decisão, através de modelos matemáticos normalmente processados computacionalmente. O principal objetivo deste trabalho é apresentar os resultados preliminares de aplicações de métodos qualitativos e quantitativos da Pesquisa Operacional no contexto da Engenharia Cartográfica e de Agrimensura, utilizando-se Mapas Conceituais, Apoio Multicritério à Decisão e Análise Envoltória de Dados.
\end{abstract}

Palavras-chave: Mapa Conceitual, Análise Multicritério à Decisão, Cartografia e Análise Envoltória de Dados.

ABSTRACT - Production Engineering dedicates to the design and management systems involving people, materials, equipment and environment. Among the sub-areas of knowledge related to Production Engineering emphasizes the Operational Research, which comes from solving real problems involving decision-making situations, using mathematical models usually processed computationally. The main objective of this paper is to present the preliminary application results of qualitative and quantity methods of Operational Research in the Surveying and Cartography Engineering using Concept Map, Multi-Criteria Evaluation and Data Envelopment Analysis (DEA)

Keywords: Concept Map, Multi-Criteria Evaluation, Cartography, Data Envelopment Analysis

\section{INTRODUÇÃO}

Métodos Formais geralmente utilizam conceitos matemáticos e notações para definir precisamente teorias e modelos de comportamento da aplicação (Bates,1996).

Conforme a ABREPO (2007 e 2008) compete à Engenharia de Produção o projeto, a implantação, a operação, a melhoria e a manutenção de sistemas produtivos integrados de bens e serviços, envolvendo homens, materiais, tecnologia, informação e energia. Compete ainda especificar, prever e avaliar os resultados obtidos destes sistemas para a sociedade e o meio ambiente, recorrendo a conhecimentos especializados da matemática, física, ciências humanas e sociais, conjuntamente com os princípios e métodos de análise e projeto da engenharia." (elaborado a partir de definições do International Institute of Industrial Engineering - IIIE e Associação Brasileira de Engenharia de Produção ABEPRO)

Vale ressaltar a multidisciplinaridade da Engenharia de Produção, uma vez que está associada às engenharias tradicionais, bem como engloba um conjunto maior de conhecimentos e habilidades, como por exemplo, Fundamentos da Administração, Gestão de Projetos, etc.

Dentre as subáreas do conhecimento relacionadas à Engenharia de Produção destaca-se a Pesquisa Operacional, que trata da resolução de problemas reais envolvendo situações de tomada de decisão, através de modelos matemáticos normalmente processados computacionalmente.

Neste trabalho serão apresentados os resultados preliminares do emprego de métodos qualitativos e quantitativos da Pesquisa Operacional, tais como Mapas Conceituais, Análise Multicritério à Decisão e Análise Envoltória de Dados, em aplicações da Engenharia Cartográfica e de Agrimensura, como, por exemplo: no monitoramento de comunidades urbanizadas, na seleção de instrumentos de automação topográfica para a realização de Levantamentos Terrestres, na escolha de alternativas para atualização cartográfica a grande escala de uma região e na avaliação da eficiência da Rede Brasileira de Monitoramento Contínuo (RBMC) do IBGE (Instituto Brasileiro de Geografia e Estatística).

O presente trabalho está organizado em 04 (quatro) seções, a saber: Introdução, Metodologia do Trabalho, 
Estudos de Caso e Conclusões. Na Seção 2 serão lançados os fundamentos básicos referentes à Mapas Conceituais, Análise Multicritério à Decisão e Análise Envoltória de Dados, objetivando a proporcionar uma visão geral sobre os métodos quantitativos e qualitativos da Pesquisa Operacional. Na Seção 3, serão apresentados os resultados preliminares de aplicações da Pesquisa Operacional no contexto da Engenharia Cartográfica e de Agrimensura. Em seguida, na Seção 4, será apresentada a conclusão do trabalho.

\section{METODOLOGIA DO TRABALHO}

A Análise Envoltória de Dados (DEA) tem sido utilizada no cálculo de indicadores de desempenho e no estabelecimento de benchmarks para regulação de setores públicos. O método presta-se ao uso em problemas multidisciplinares e multiagentes, podendo ser utilizado na estimação de funções da fronteira de produção ou incorporando a opinião de especialistas, tal como um método multicritério.

Em DEA, segundo Kassai (2002), as unidades produtivas homogêneas denominadas DMU ("Decision Making Unit") devem ser comparáveis e atuar sobre as mesmas condições, se diferenciando apenas na intensidade ou magnitude. Dessa forma é imprescindível conhecer o comportamento das referidas unidades, bem como a homogeneidade dos dados. Conforme Ceretta e Niedearauer (2001) e Badin (1997) diferentes ordens de grandeza em DEA podem ser tratadas através do agrupamento, também conhecido como análise de cluster.

Embora existam várias formas de construção das fronteiras de produção de unidades produtivas (DMU), podem-se destacar dois modelos que são considerados clássicos: o CCR e o BCC. O primeiro também conhecido como modelo CRS (Constant Returns to Scale) foi apresentado originalmente por Charnes et al. (1978) e trabalha com retornos constantes de escala, isto é, qualquer variação nas entradas (input) produz variação proporcional nas saídas (output). O segundo também conhecido como VRS (Variable Returns to Scale) foi introduzido por Banker et al. (1984), considera retornos variáveis de escala e substitui o axioma da proporcionalidade entre input e output pelo da convexidade.

Por definição, há 2 (duas) formulações empregadas nos Modelos DEA, quais sejam: Envelope, que define uma região viável de produção e trabalha com uma projeção de cada DMU na fronteira desta região; e Multiplicadores, que emprega a razão das somas ponderadas de produtos e recursos. No que tange à orientação, busca-se a fronteira eficiente minimizando as entradas e mantendo constante o nível de saídas observadas; ou maximizando as saídas e mantendo constante o nível de entradas observadas. Tais orientações são denominadas, respectivamente, a insumo e a produto.

Os modelos clássicos de DEA assumem que os inputs devem ser minimizados e os outputs maximizados. Todavia, o processo produtivo pode gerar resultados (outputs) indesejáveis. Várias abordagens para equacionar esse tipo de problema vêm sendo produzidas, como por exemplo, a transformação multiplicativa inversa (MLT), que foi proposta por Golany e Roll (1989) e é frequentemente usada. A técnica consiste em calcular o valor inverso de cada output indesejado, transformando-o em "output" desejado.

O conceito de índice de produtividade de Malmquist, de acordo com Sollero (2007), foi introduzido por Malmquist em 1953, sendo que Färe (1992) forneceu a fundamentação para construir o índice de Malmquist empregando técnicas de programação linear.

Há diversas aplicações práticas do referido índice, dentre as quais se podem destacar nas seguintes áreas do conhecimento: Saúde (Lobo et al., 2009), Portuária (Kirchner,2013), Educação (Agasisti; Pérez-Esparrells, 2010), Agricultura (Coelli;Rao,2003) Energia (Wu et al., 2013), Finanças (Asmild et al., 2004), dentre outras.

$O$ Índice de Malmquist avalia os índices de produtividade em diferentes períodos de tempo, decompondo-os em subíndices que refletem variação da eficiência técnica e mudanças tecnológicas. Esta decomposição do índice de Malmquist contribui para uma análise das alterações nos índices de produtividade, pois permite identificar se um aumento é fruto do progresso tecnológico ou da melhoria na eficiência técnica, ou ainda, dos dois simultaneamente.

$\mathrm{O}$ Índice de Malmquist $\left(\mathrm{M}_{\mathrm{o}}\right)$, que corresponde à medida do crescimento da produtividade total dos fatores (Total Factor Productivity - TFP Growth), permite acompanhar a evolução da produtividade das unidades avaliadas ao longo dos anos por intermédio da medição da variação de produtividade entre os períodos $t$ e $t+1$. $O$ referido índice é calculado pela seguinte fórmula (Equação 1):

$$
M_{o}=\sqrt[2]{\frac{\theta_{o}^{t}\left(x_{o}^{t}, y_{o}^{t}\right)}{\theta_{o}^{t}\left(x_{o}^{t+1}, y_{o}^{t+1}\right)} \frac{\theta_{o}^{t+1}\left(x_{o}^{t}, y_{o}^{t}\right)}{\theta_{o}^{t+1}\left(x_{o}^{t+1}, y_{o}^{t+1}\right)}}
$$

Onde:

$\theta_{o}^{t}\left(x_{o}^{t}, y_{o}^{t}\right):$ Corresponde à medida de eficiência técnica da $\mathrm{DMU}_{0}$ no período $\mathrm{t}$, obtida com as observações de todas as DMU no período t;

$\theta_{o}^{t+1}\left(x_{o}^{t+1}, y_{o}^{t+1}\right):$ Corresponde à medida de eficiência técnica da $\mathrm{DMU}_{0}$ no período $\mathrm{t}+1$, obtida com as observações de todas as DMU no período $\mathrm{t}+1$;

$\theta_{o}^{t}\left(x_{o}^{t+1}, y_{o}^{t+1}\right)$ : Corresponde à medida de eficiência técnica da $\mathrm{DMU}_{0}$ obtida ao substituir os dados da $\mathrm{DMU}_{0}$ no período $\mathrm{t}$ por aqueles do período $\mathrm{t}+1$, desde que as observações das demais DMU tenham sido realizadas no período t; e

$\theta_{o}^{t+1}\left(x_{o}^{t}, y_{o}^{t}\right)$ : Corresponde à medida de eficiência técnica da $\mathrm{DMU}_{0}$ obtida ao substituir os dados da $\mathrm{DMU}_{0}$ no período $t+1$ por aqueles do período $t$, desde que as observações das demais DMU tenham sido realizadas no período $\mathrm{t}+1$. 
Se o Mo > 1 há declínios de produtividade, se o Mo $=1$ permanece inalterado e, finalmente há melhora se o Mo $<1$.

De acordo com Gomes et al (2006), Apoio Multicritério à Decisão é uma área dinâmica do conhecimento e da pesquisa, orientada para apoiar os decisores e os negociadores, auxiliando na estruturação dos problemas, permitindo expandir a argumentação e ampliando a capacidade de aprendizagem e compreensão, objetivando a auxiliar o decisor (ou decisores) durante o processo de decisão.

Segundo Bana e Costa, (apud Detoni, 1996), o apoio à decisão é a atividade daquele que, servindo-se de modelos claramente explicitados e mais ou menos formalizados, procura obter elementos de resposta às questões a um interveniente num processo de decisão, elementos esses concorrentes a esclarecer a decisão e normalmente a recomendar, ou simplesmente a favorecer, um comportamento de natureza a aumentar a coerência entre a evolução do processo por um lado, os objetivos e os sistemas de valores desse interveniente por outro lado.

As metodologias multicritérios de apoio à decisão apresentam conceitos e bases para estruturar e modelar uma situação problemática, incluindo maneiras de identificar e gerar ações e técnicas de construção de critérios e procedimentos para agregação dos mesmos (Bana e Costa et al., 1996a).

Na perspectiva de apoio à decisão, a consideração da subjetividade, além da objetividade, dos elementos na construção de modelos de avaliação, constitui uma das principais vantagens dos atuais modelos multicritérios sobre os modelos clássicos de pesquisa operacional (Bana e Costa et al., 1996b).

Segundo Roy e Bouyssou (1993), as problemáticas básicas de apoio à decisão são as seguintes:

- Seleção: o objetivo consiste em esclarecer a decisão pela escolha de um subconjunto, tão restrito quanto possível, tendo em vista a escolha final de uma única ação. Esse subconjunto conterá as melhores alternativas ou as alternativas satisfatórias. $\mathrm{O}$ resultado do processo será uma escolha ou um procedimento de seleção;

- Triagem: o objetivo é esclarecer a decisão por uma triagem resultante de uma alocação de cada alternativa a uma categoria ou classe. As categorias são definidas a priori a partir de normas aplicáveis ao conjunto de alternativas. O resultado do processo será uma triagem ou um procedimento de alocação;

- Ordenação: o objetivo é esclarecer a decisão por um arranjo obtido reagrupando-se todas ou parte (as mais satisfatórias) das alternativas em classes de equivalência, estas classes sendo ordenadas de modo completo ou parcial, conforme as preferências. O resultado do processo de decisão será um arranjo ou um procedimento de classificação; e

- Descrição: o objetivo consiste em esclarecer a decisão por uma descrição, em uma linguagem apropriada, das alternativas e de suas consequências. $\mathrm{O}$ resultado do processo será uma descrição ou um procedimento cognitivo.

Há duas escolas de Apoio Multicritério a Decisão, quais sejam: a Escola Europeia e a Escola Americana. A primeira é representada principalmente pelos métodos Elimination and Choice Expressing Reality (ELECTRE) e Preference Ranking Organization Method for Enrichment Evaluations (PROMETHEE), enquanto que a segunda pelo Analytic Hierarchy Process (AHP).

Roy e Vanderpooten (1995) mencionam que a Escola Europeia possui a tendência de que as preferências dos decisores influenciem menos nas escolhas das alternativas, ao contrário da Americana.

Segundo Acolet (2008), a Escola Europeia considera, ainda, a incomparabilidade entre duas alternativas, não impondo a necessidade de hierarquização das alternativas e não havendo necessariamente função matemática para definição de valor das alternativas.

Muito embora não tenha sido objeto dos Estudos de Caso apresentados no item 3 (três) deste trabalho, vale ressaltar que há inúmeras aplicações da Análise Multicritério na Engenharia Cartográfica e de Agrimensura, no que tange à elaboração de Mapas Temáticos, que são destinados a um tema específico, necessários às pesquisas socioeconômicas, de recursos naturais e de estudos ambientais.

O Método de Análise Hierárquica foi um dos primeiros Métodos de Apoio Multicritério à Decisão sendo amplamente utilizado em todo o mundo atualmente. O método se caracteriza pela decomposição de um problema discreto em uma estrutura hierárquica descendente, iniciando pelo objetivo global, critérios, subcritérios e alternativas em níveis sucessivos (Ribeiro et al., 2007 apud Saaty 1990). Assim, o método propicia ao decisor uma melhor avaliação e compreensão do problema de decisão (Gomes et al, 2004). Posteriormente à construção da hierarquia, é realizada a modelagem, na qual, por meio de comparações pareadas e do julgamento do decisor, as alternativas serão priorizadas.

O método multicritério de análise de decisão denominado AHP - Analytic Hierarchy Process foi desenvolvido por Thomas L. Saaty em meados da década de 1970 no intuito de promover a superação das limitações cognitivas dos tomadores de decisão. É aplicado para sistematizar uma ampla gama de problemas de decisão nos contextos: econômico, político, social e ambiental, devido a sua simplicidade, robustez e capacidade de avaliar fatores qualitativos e quantitativos sejam eles tangíveis ou intangíveis (SHIAU et al., 2002). Existem no mercado alguns programas, como AUTOMAN, Criterium, Expert Choice, HIPRE3+ e NCIC que implementam o AHP. Dentre eles, o mais utilizado é o Expert Choice, entretanto o preço da licença comercial é elevado.

Outra opção para implementar o uso do AHP é o software Criterium. O Criterium DecisionPlus (CDP) é uma versátil ferramenta computacional que ajuda os usuários a fazer complexas decisões entre alternativas envolvendo múltiplos critérios. Além dos aspectos 
positivos citados, o programa possui uma versão gratuita, restrita ao uso acadêmico.

Segundo Rosenhead e Mingers (2001), a estruturação de problemas é uma das etapas do processo de tomada de decisão que tem como objetivo estruturar assuntos, problemas e situações para os quais se buscam propostas de decisões e não apenas resolver uma parte simplificada do problema sob uma perspectiva em particular.

De acordo com Okada, Buckingham e Sherborne (2008), os mapas conceituais são ferramentas gráficas utilizadas para representação do conhecimento, de modo que dois conceitos podem ser ligados através de uma frase de ligação, gerando desta forma uma proposição. Novak (1998) apresenta os mapas conceituais como ferramenta útil ao aprendizado de alunos dos diversos níveis, bem como no auxílio na solução de problemas em organizações.

Considera-se que uma representação gráfica é mais efetiva que um texto para a comunicação de conteúdos complexos porque o processamento mental das imagens pode ser menos exigente cognitivamente que o processamento verbal de um texto (Vekiri, 2002).

Neste trabalho será apresentada, também, uma abordagem estratégica para a formulação e estruturação do caso em estudo, por intermédio do uso de mapa conceitual. Vale lembrar que diretrizes sobre a elaboração de mapas conceituais podem ser encontradas na literatura, como em Moreira (2006) e Ruiz-Moreno et al (2007).

É necessário ressaltar, ainda, que a tomada de decisão é uma função humana. O papel do modelo é apenas auxiliar os especialistas, garantindo qualidade, organização e documentação do processo de análise de decisão, explicitando valores de julgamentos, analisando objetivos conflitantes, compartilhando o entendimento sobre as questões e, frequentemente, promovendo consenso

\section{ESTUDOS DE CASO}

Em Antoun Netto et al. (2013) é apresentado o emprego de ortofotos digitais e Índice de Malmquist no monitoramento de comunidades urbanizadas no município do Rio de Janeiro $\mathrm{Na}$ Tab. 1, serão apresentados o Índice de Malmquist das 31(trinta e uma) Comunidades Urbanizadas, que apresentam área ocupada maior que $100000 \mathrm{~m}^{2}$ (cem mil metros quadrados), entre dois períodos de tempo, quais sejam: 1999 e 2010, a fim de que seja avaliada a variação da produtividade, no que tange à população e à área ocupada das referidas comunidades.

Tabela 1 - Relatório do Software DEAFrontier para o Índice de Malmquist (1999/2010)

\begin{tabular}{|c|c|c|c|c|}
\hline$D M U$ & Name & $\begin{array}{c}\text { Output-Oriented } \\
\text { CRS } \\
\text { Malmquist Index }\end{array}$ & $\begin{array}{c}\text { Efficiency } \\
\text { Change }\end{array}$ & $\begin{array}{c}\text { Frontier } \\
\text { Shift }\end{array}$ \\
\hline 1 & Arrelia & 0,44703 & 0,34179 & 1,30790 \\
\hline 2 & Bairro Agulhas Negras & 0,72200 & 0,55203 & 1,30790 \\
\hline 3 & Bairro Nova Aliança & 0,51984 & 0,39746 & 1,30790 \\
\hline 4 & Baixa do Sapateiro & 1,30994 & 1,00156 & 1,30790 \\
\hline 5 & Borel & 0,94705 & 0,72409 & 1,30790 \\
\hline 6 & Caminho do Lúcio & 1,00122 & 0,76552 & 1,30790 \\
\hline 7 & Divinéia & 1,57215 & 1,20204 & 1,30790 \\
\hline 8 & Faz quem Quer & 0,74607 & 0,57043 & 1,30790 \\
\hline 9 & Fazenda Botafogo & 1,00635 & 0,76943 & 1,30790 \\
\hline 10 & Floresta da Barra da Tijuca & 1,01236 & 0,77403 & 1,30790 \\
\hline 11 & Jamelão & 1,31724 & 1,00714 & 1,30790 \\
\hline 12 & Jardim Moriçaba & 0,80207 & 0,61325 & 1,30790 \\
\hline 13 & Mangueira & 0,76521 & 0,58506 & 1,30790 \\
\hline 14 & Morro da Formiga & 1,27098 & 0,97177 & 1,30790 \\
\hline 15 & Morro dos Telégrafos & 0,66584 & 0,50909 & 1,30790 \\
\hline 16 & Morro da União & 6,99084 & 5,34507 & 1,30790 \\
\hline 17 & Nova Holanda & 0,97353 & 0,74434 & 1,30790 \\
\hline 18 & Parque Acari & 1,01298 & 0,77451 & 1,30790 \\
\hline 19 & Parque Boa Esperança & 0,34306 & 0,26230 & 1,30790 \\
\hline 20 & Parque Jardim Beira Mar & 0,92422 & 0,70664 & 1,30790 \\
\hline 21 & Parque Maré & 1,02817 & 0,78612 & 1,30790 \\
\hline 22 & Parque Proletário de Vigário Geral & 1,06141 & 0,81153 & 1,30790 \\
\hline 23 & Parque União & 0,62189 & 0,47549 & 1,30790 \\
\hline 24 & Salgueiro & 1,05569 & 0,80716 & 1,30790 \\
\hline 25 & Santa Maria & 0,85371 & 0,65273 & 1,30790 \\
\hline 26 & São Jerônimo & 0,75082 & 0,57407 & 1,30790 \\
\hline 27 & Timbau & 0,94673 & 0,72385 & 1,30790 \\
\hline 28 & Três Pontes & 0,87987 & 0,67273 & 1,30790 \\
\hline 29 & Vidigal & 0,96229 & 0,73575 & 1,30790 \\
\hline 30 & Vila do Céu & 0,85899 & 0,65677 & 1,30790 \\
\hline 31 & Vila São Jorge & 1,05456 & 0,80630 & 1,30790 \\
\hline
\end{tabular}


Verifica-se que, em aproximadamente 61\% (sessenta e um por cento) das comunidades consideradas, houve uma melhora de eficiência nos períodos avaliados, entretanto a média geral do Índice de Malmquist para as 31 (trinta e uma) comunidades corresponde a 1,1105, que aponta uma piora na eficiência nos períodos considerados.

Em Antoun Netto e Vasconcellos (2013) é apresentada uma aplicação da Análise de Decisão Multicritério na seleção de instrumentos de automação topográfica. O mapa conceitual constante da Fig. 1 foi o resultado do emprego da abordagem estratégica para a formulação e estruturação do caso em estudo da seleção de instrumento de automação topográfica

Neste trabalho foi utilizada a ferramenta computacional CMAPTOOLS, versão 4.11, desenvolvida pelo "Institute for Human and Machine Cognition (IHMC) da UWF - University of West Florida", que permite aos usuários construir, navegar, compartilhar e criticar modelos de conhecimento representados como mapas conceituais.

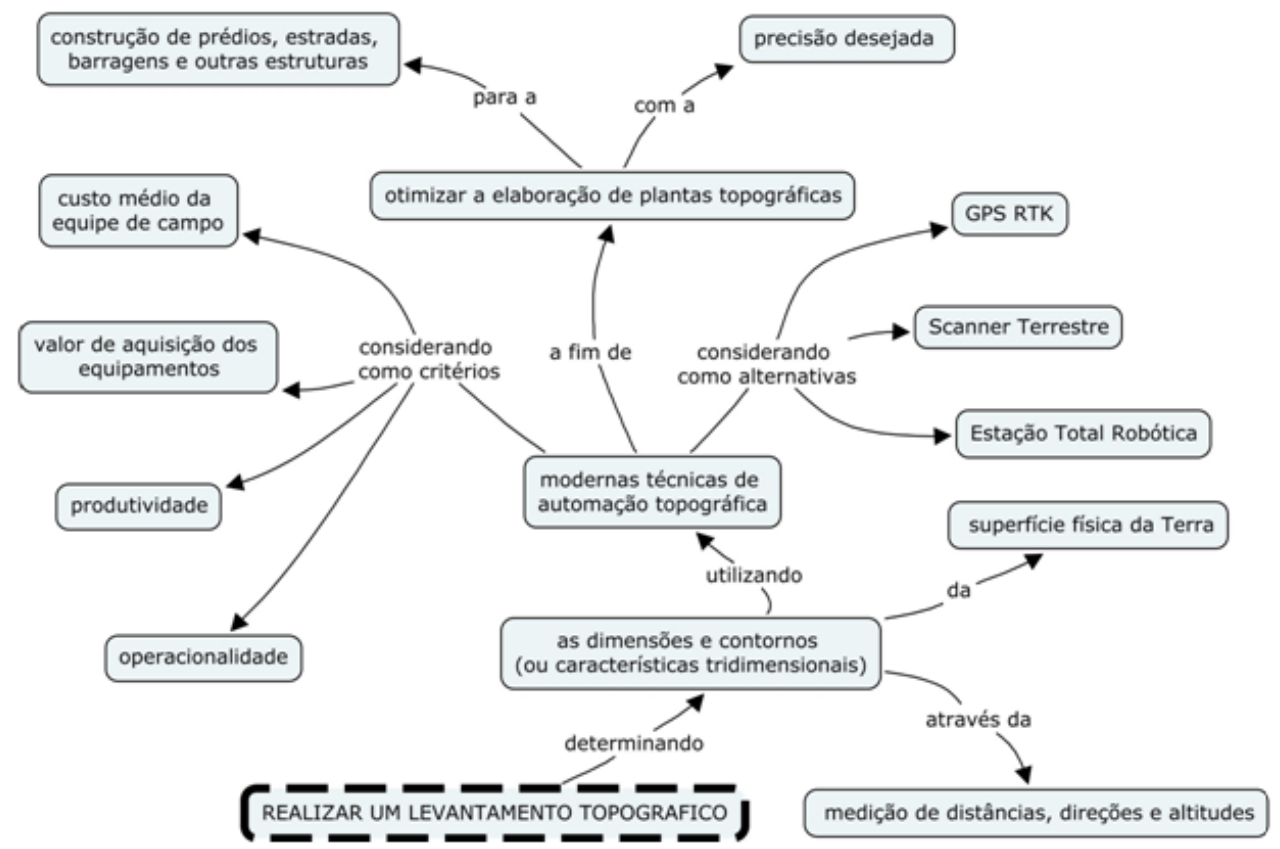

Figura 1 - Mapa Conceitual para a seleção de instrumentos de automação topográfica

A partir do mapa conceitual, foi possível determinar o conjunto de ações possíveis (alternativas) do estudo em causa, conforme abaixo especificado:

$\checkmark$ GPS RTK (Real Time Kinematic);

$\checkmark$ Scanner terrestre; e

$\checkmark$ Estação Total Robótica.

Determinou-se, ainda, a família de critérios a serem utilizados com as suas respectivas escalas qualitativas, conforme apresentado na Tab. 2, onde:

$\checkmark$ Operacionalidade $(\mathrm{O})$ : corresponde a facilidade de operação no campo considerando-se os fatores, tais como: topografia do terreno (plano ou acidentado), obstrução do sinal em áreas com vegetação, edificações, veículos estacionados, etc.

$\checkmark$ Investimento (I): Valor de aquisição dos equipamentos em reais $(\mathrm{R} \$)$;

$\checkmark$ Produtividade (P): Quantidade de pontos levantados e tempo de levantamento; $\mathrm{e}$

$\checkmark$ Custo (C): Custo médio da equipe de campo em reais $(\mathrm{R} \$)$.
Tabela 2 - Família de Critérios

\begin{tabular}{|l|l|}
\hline \multicolumn{1}{|c|}{ Critério } & Escala Quantitativa (*) \\
\hline Investimento $(\mathrm{I})$ & \\
\cline { 1 - 1 } Custo $(\mathrm{C})$ & 1 a 5 \\
\cline { 1 - 1 } Operacionalidade $(\mathrm{O})$ & \\
\cline { 1 - 1 } Produtividade $(\mathrm{P})$ & \\
\hline
\end{tabular}

(*) 1 é a pior situação e 5 , a melhor.

A seguir, é apresentado na Tab. 3 o Quadro de Preferências da situação problema, que será uma ferramenta imprescindível para a implementação da referida situação nos diversos métodos de apoio multicritério à decisão. Cumpre salientar que o tomador de decisão da ação em estudo é o Engenheiro encarregado do levantamento topográfico

Tabela 3 - Quadro de Preferências do caso em estudo

\begin{tabular}{|l|l|l|l|l|}
\cline { 2 - 5 } \multicolumn{1}{c|}{} & I & C & P & O \\
\hline GPS RTK & 3 & 3 & 2 & 3 \\
\hline Scanner Terrestre & 1 & 5 & 5 & 1 \\
\hline Estação Total Robótica & 5 & 1 & 1 & 5 \\
\hline
\end{tabular}

O software Criterium Decision Plus (CDP) foi escolhido objetivando a apresentar a alternativa mais viável para a solução da situação problema. Na Fig. 2 será apresentado o resultado consolidado do ranking das 
alternativas no método AHP (software CDP) nos diferentes cenários considerados neste trabalho, quais sejam:

a. Atribuição de importância ao critério custo e de irrelevância aos demais critérios;

b. Atribuição de importância ao critério produtividade e de irrelevância aos demais critérios; c. Atribuição de importância ao critério operacionalidade e de irrelevância aos demais critérios; e

d. Atribuição de importância ao critério investimento e de irrelevância aos demais critérios

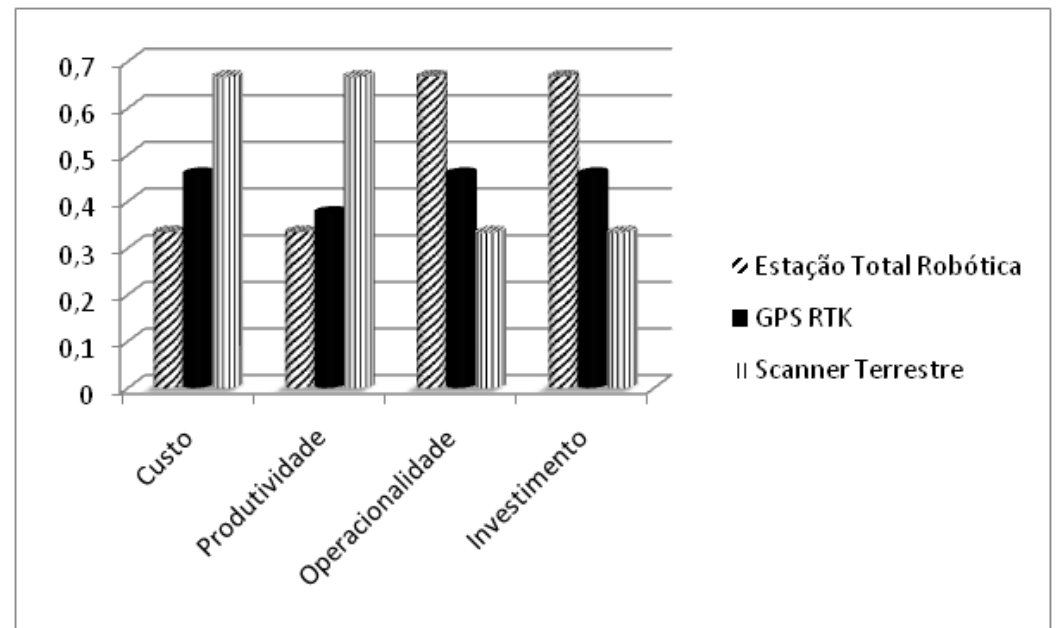

Figura 2 - Resultado consolidado do ranking das alternativas no método AHP

Em Antoun Netto et al. (2011) é apresentada uma proposta de seleção de alternativas para atualização cartográfica a grande escala através do apoio multicritério à decisão.
Inicialmente, foi realizada a estruturação do caso em estudo, por intermédio do uso de mapa conceitual, conforme apresentado na Fig. 3.

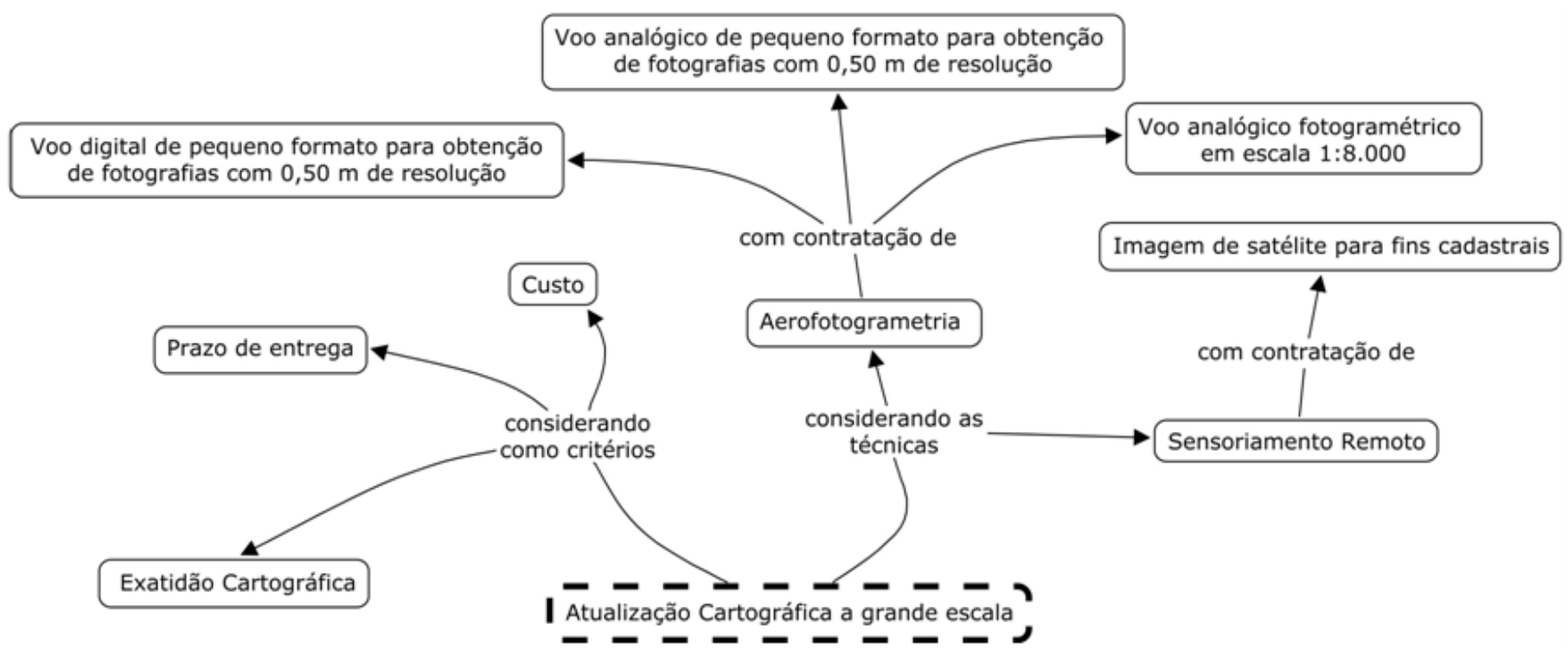

Figura 3 - Mapa Conceitual da Atualização Cartográfica a grande escala

Para a elaboração do Quadro de Preferências foram consideradas as premissas a seguir relacionadas:

$\checkmark$ Possibilidade de Pontuação: 1, 2, 3, 4, 5; onde 1 é a pior situação e 5, a melhor; $\checkmark$ Os valores da pontuação dos diversos critérios foram arbitrados pelo autor;

$\checkmark$ Será utilizada hipoteticamente a escala de 1:2.000 para a representação cartográfica do núcleo central urbano; e 
$\checkmark \quad$ O prazo de entrega corresponde ao tempo médio transcorrido no processo de geração de um documento cartográfico, contando desde a coleta da informação superficial.
A seguir, é apresentado, na Tab. 4, o Quadro de Preferências da situação problema, que será uma ferramenta imprescindível para a implementação da referida situação no Método AHP de apoio multicritério à decisão.

Tabela 4 - Quadro de Preferências do Caso em Estudo

\begin{tabular}{|l|c|c|c|}
\hline \multicolumn{1}{|c|}{ Ações Possíveis } & \multicolumn{3}{|c|}{ Critérios } \\
\cline { 2 - 4 } & Custo (\$) & $\begin{array}{c}\text { Prazo de } \\
\text { Entrega }\end{array}$ & $\begin{array}{c}\text { Exatidão } \\
\text { Cartográfica }\end{array}$ \\
\hline $\begin{array}{l}\text { Voo digital de pequeno formato } \\
\text { para obtenção de fotografias } \\
\text { com 0,50 de resolução }\end{array}$ & 4 & 1 & 4 \\
\hline $\begin{array}{l}\text { Voo analógico de pequeno } \\
\text { formato para obtenção de } \\
\text { fotografias com 0,50 m de } \\
\text { resolução }\end{array}$ & 3 & 1 & 4 \\
\hline $\begin{array}{l}\text { Voo analógico fotogramétrico } \\
\text { em escala 1:8.000 }\end{array}$ & 1 & 2 & 5 \\
\hline $\begin{array}{l}\text { Imagem de satélite para fins } \\
\text { cadastrais }\end{array}$ & 5 & 5 & 1 \\
\hline
\end{tabular}

Na Fig. 4 será apresentado o resultado consolidado do ranking das alternativas no método AHP (software CDP) sem atribuição de pesos e com peso maior nos critérios prazo de entrega, exatidão cartográfica e custo, respectivamente.

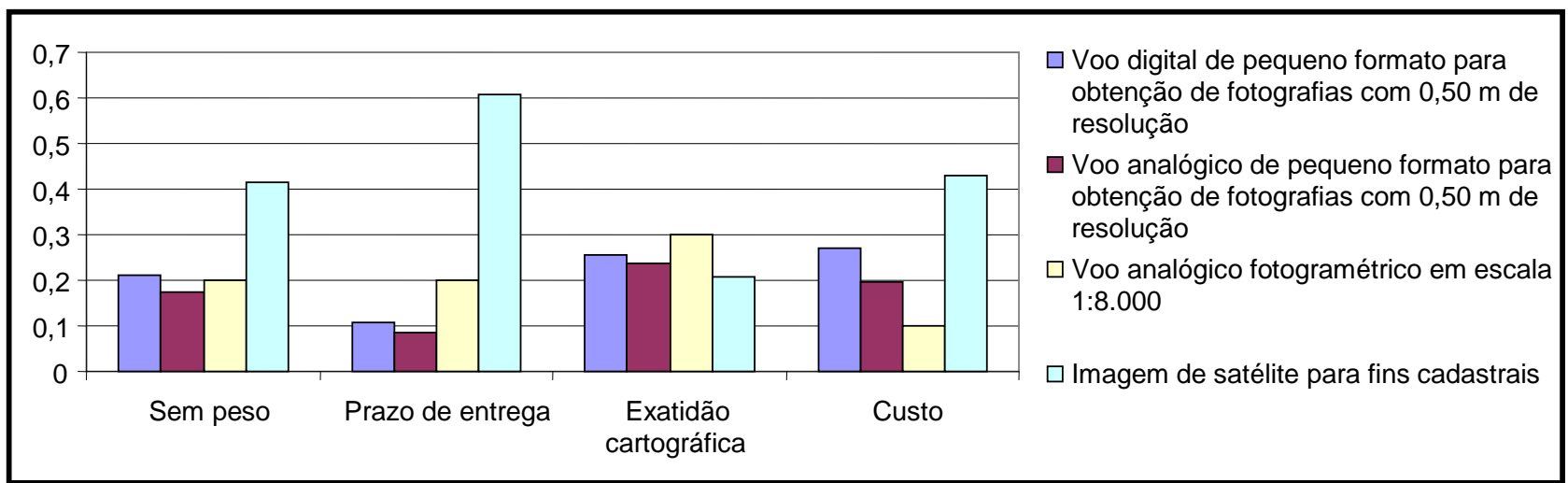

Figura 4 - Resultado consolidado do ranking das alternativas no método AHP

Em Antoun Netto e Vasconcellos (2013) é proposta uma metodologia para a avaliação da eficiência da Rede Brasileira de Monitoramento Contínuo (RBMC) com o uso da Análise Envoltória de Dados (DEA).Na Tab. 5 são apresentadas as variáveis a serem consideradas dentro do modelo. A escolha de variáveis em DEA é uma questão chave para a determinação da eficiência.

Tabela 5 - Variáveis identificadas no Estudo de Caso

\begin{tabular}{|c|l|}
\hline \multirow{2}{*}{ INPUT } & Erro Médio Quadrático devido ao Multicaminho em L1 (MP1) \\
\cline { 2 - 3 } & Erro Médio Quadrático devido ao Multicaminho em L2 (MP2) \\
\hline \multirow{2}{*}{ OUTPUT } & Número de Dias com a Estação em Operação por ano \\
\cline { 2 - 3 } & Erro Médio Quadrático da Relação Sinal-Ruído em L1 por dia (SNR1) \\
\cline { 2 - 3 } & Erro Médio Quadrático da Relação Sinal-Ruído em L2 por dia (SNR2) \\
\hline
\end{tabular}

Neste trabalho, a fim de minimizar possíveis anomalias no modelo DEA foram utilizadas as variáveis Erro Médio Quadrático devido ao Multicaminho em L1 (MP1) e Erro Médio Quadrático devido ao Multicaminho em L2 (MP2) como de entrada (input), não sendo empregada a transformação multiplicativa inversa (MLT).

Decidiu-se por assumir o tipo de fronteira VRS (Variable Returns to Scale) orientado a output, a ser utilizado na modelagem DEA sem introdução de restrições aos pesos. Tal fronteira considera a possibilidade de rendimentos crescentes ou decrescentes de escala na fronteira eficiente.

$\mathrm{Na}$ Tab. 6, serão apresentadas as estações RBMC eficientes obtidas por intermédio do software 
DEAFrontier, desenvolvido pelo Professor Joe Zhu da WPI (Worcester Polytechnic Institute).

Tabela 6 - Estações RBMC eficientes

\begin{tabular}{|l|l|}
\hline \multirow{5}{*}{ Benchmark } & ALAR, BRAZ, CHPI, CRUZ, ILHA, \\
& MCLA, MGBH, MGVA, MTBA, \\
& ONRJ, POLI, PPTE, SCLA, SPCA, \\
SSA1 e UBER
\end{tabular}

Tabela 7 - Ranking das estações RBMC (2012)

\begin{tabular}{|c|c|c|}
\hline Ranking & Estação & Eficiência \\
\hline 1 & $\begin{array}{l}\text { ALAR, BRAZ, CHPI, CRUZ, ILHA, } \\
\text { MCLA, MGBH, MGVA, MTBA, } \\
\text { ONRJ, POLI, PPTE, SCLA, SPCA, } \\
\text { SSA1 e UBER }\end{array}$ & 1 \\
\hline 2 & NEIA & 0,9997 \\
\hline 3 & BRFT & 0,9989 \\
\hline 4 & UFPR & 0,9989 \\
\hline 5 & POAL & 0,9973 \\
\hline 6 & SALU & 0,9969 \\
\hline 7 & CUIB & 0,9933 \\
\hline 8 & IMPZ & 0,9930 \\
\hline 9 & IMBT & 0,9919 \\
\hline 10 & BAVC & 0,9913 \\
\hline 11 & RIOD & 0,9910 \\
\hline 12 & MAPA & 0,9897 \\
\hline 13 & ROJI & 0,9888 \\
\hline 14 & BAIR & 0,9887 \\
\hline 15 & BOMJ & 0,9881 \\
\hline 16 & SAVO & 0,9871 \\
\hline 17 & ROGM & 0,9840 \\
\hline 18 & SPBO & 0,9792 \\
\hline 19 & MGMC & 0,9782 \\
\hline 20 & POVE & 0,9776 \\
\hline 21 & PSIR & 0,9776 \\
\hline 22 & RNNA & 0,9754 \\
\hline 23 & RNMO & 0,9746 \\
\hline 24 & RJCG & 0,9730 \\
\hline 25 & GOJA & 0,9726 \\
\hline 26 & SAGA & 0,9711 \\
\hline 27 & SMAR & 0,9708 \\
\hline 28 & PRGU & 0,9682 \\
\hline 29 & MGUB & 0,9675 \\
\hline 30 & MGIN & 0,9674 \\
\hline 31 & PITN & 0,9671 \\
\hline 32 & ROCD & 0,9671 \\
\hline 33 & TOPL & 0,9669 \\
\hline 34 & PBCG & 0,9666 \\
\hline
\end{tabular}

Verificou-se no relatório do referido software que não há ocorrência de estações RBMC eficientes na Região Pareto-Ineficiente, ou seja, as referidas estações não apresentam folgas nas variáveis de input e output e os benchmarks das mesmas são elas próprias, não sendo
A seguir, na Tab. 7 será apresentado o ranking final das eficiências em ordem decrescente das estações RBMC avaliadas.

\begin{tabular}{|c|c|c|}
\hline Ranking & Estação & Eficiência \\
\hline 35 & ROSA & 0,9665 \\
\hline 36 & PEPE & 0,9663 \\
\hline 37 & VICO & 0,9661 \\
\hline 38 & PRMA & 0,9659 \\
\hline 39 & MTCO & 0,9645 \\
\hline 40 & GVAL & 0,9642 \\
\hline 41 & SCCH & 0,9633 \\
\hline 42 & MSCG & 0,9591 \\
\hline 43 & BATF & 0,9557 \\
\hline 44 & CRAT & 0,9551 \\
\hline 45 & BELE & 0,9547 \\
\hline 46 & PAAT & 0,9529 \\
\hline 47 & MTCN & 0,9503 \\
\hline 48 & BOAV & 0,9489 \\
\hline 49 & OURI & 0,9471 \\
\hline 50 & MTSF & 0,9449 \\
\hline 51 & SPAR & 0,9425 \\
\hline 52 & CEFT & 0,9388 \\
\hline 53 & TOGU & 0,9368 \\
\hline 54 & SJRP & 0,9358 \\
\hline 55 & MGRP & 0,9349 \\
\hline 56 & SPJA & 0,9331 \\
\hline 57 & NAUS & 0,9323 \\
\hline 58 & CEEU & 0,9270 \\
\hline 59 & UBA1 & 0,9267 \\
\hline 60 & BABR & 0,9260 \\
\hline 61 & AMCO & 0,9074 \\
\hline 62 & MABA & 0,8941 \\
\hline 63 & PAIT & 0,8926 \\
\hline 64 & MABS & 0,8270 \\
\hline 65 & APSA & 0,8178 \\
\hline & & \\
\hline 50 & \\
\hline 50
\end{tabular}

necessária a aplicação do conceito de Russell a um alvo obtido em um modelo DEA clássico para equacionar tal ocorrência. 


\section{CONCLUSÃO}

Não se pretende nesse trabalho noticiar os resultados obtidos como modelo final do desempenho, uma vez que a determinação das variáveis e a introdução de restrições aos pesos devem ser definidas, após amplo debate, por meio de consenso com todos os atores envolvidos no processo.

Assim sendo, almeja-se que os resultados apresentados sejam compatíveis com as novas tendências de aplicação de métodos quantitativos e qualitativos da Pesquisa Operacional.

Pode-se indicar que as eficiências calculadas por DEA são relativas, isto é, as eficiências são calculadas com relação aos dados observados e em comparação às outras DMU, portanto uma DMU eficiente pode incrementar seu desempenho o que determinaria uma nova fronteira de produção.

No que se refere às ineficiências obtidas e à determinação dos valores ótimos, permitem aos gestores localizar as fontes de ineficiência, possibilitando identificar ações para reduzi-las. Cumpre ressaltar que os julgamentos realizados para construção do Quadro de Preferências dos Casos em Estudo foram contribuições pessoais dos autores, baseadas nas suas percepções, experiências, interesses e conhecimentos. Possivelmente, os resultados poderiam ser melhores na hipótese da participação de pelo menos mais dois especialistas nas áreas de Topografia, Fotogrametria e Sensoriamento Remoto no procedimento de obtenção dos julgamentos de valor.

Pode-se depreender que os resultados obtidos deram embasamento à metodologia proposta que visa à aplicação de métodos formais da Engenharia de Produção na Engenharia Cartográfica e de Agrimensura, utilizandose a conjugação das metodologias "soft" e "hard" da Pesquisa Operacional.

\section{REFERÊNCIAS BIBLIOGRÁFICAS}

ABEPRO Associação Brasileira de Engenharia de Produção. Engenharia de Produção: Grande Área e Diretrizes Curriculares XVII Encontro Nacional de Engenharia de Produção, 1997.

ABEPRO Associação Brasileira de Engenharia de Produção. Engenharia de Produção: Grande Área e Diretrizes Curriculares III Encontro de Coordenadores de Cursos de Engenharia de Produção (III ENCEP), Itajubá, 1998.

ACOLET, TATIANA. Modelo de análise de crédito fundamentado no ELECTRE TRI. Rio de Janeiro: Faculdades Ibmec. Dissertação de Mestrado Profissionalizante apresentada ao Programa de PósGraduação em Administração das Faculdades Ibmec, 2008 .

AGASISTI T, PÉREZ-ESPARRELLS C. Comparing efficiency in a cross-country perspective: the case of
Italian and Spanish state universities. High Educ 59(1):85-103, 2010.

ANTOUN NETTO, S. O. ; VASCONCELLOS, J.C.P.D.; Destri, A. R.. Ortofotos digitais e Índice de Malmquist aplicados ao monitoramento de comunidades urbanizadas no município do Rio de Janeiro. In: XVI Simpósio Brasileiro de Sensoriamento Remoto SBSR, 2013, Foz do Iguaçu. Anais do $16^{\circ}$ Simpósio Brasileiro de Sensoriamento Remoto, 2013. v. 1. p. 1114-1120.

ANTOUN NETTO, S. O. ; LINS, MARCOS PEREIRA ESTELLITA; VASCONCELLOS, J.C.P.D. Seleção de alternativas para atualização cartográfica a grande escala através do Apoio Multicritério à Decisão. In: XXV Congresso Brasileiro de Cartografia, 2011, Curitiba- PR. Anais do XXV Congresso Brasileiro de Cartografia. Rio de Janeiro: Sociedade Brasileira de Cartografia, 2011. p. 118-123.

ANTOUN NETTO, S. O.; VASCONCELLOS, J.C.P.D. A proposal for evaluating RBMC (Brazilian Network for Continuous Monitoring) using Data Envelopment Analysis (DEA). 26th International Cartographic Conference (ICC 2013) August 25 -30, 2013 Dresden, Germany.

ANTOUN NETTO, S. O. ; VASCONCELLOS, J.C.P.D.. Análise de decisão multicritério aplicada na seleção de instrumentos de automação topográfica. 2013. (Apresentação de Trabalho/Simpósio). XLV SBPO Simpósio Brasileiro de Pesquisa Operacional, realizado em Natal $(\mathrm{RN})$ de 16 a 19 de setembro de 2013.

ASMILD, M., PARADI, C.V., AGGARWALL, V., SCHAFFNIT, C.,. Combining DEA window analysis with the Malmquist index approach in a study of the Canadian banking industry. Journal of Productivity Analysis, 21, 67-89, 2004.

BADIN, N. T. Avaliação da produtividade de supermercados e seu benchmarking. Dissertação Programa de Pós-Graduação em Engenharia de Produção, Universidade Federal de Santa Catarina, Florianópolis, 1997.

CERETTA, P.S.; NIEDERAUER, C.A.P. Rentabilidade e eficiência no setor bancário brasileiro. RAC, v.5, n.3, set/dez, p.7-26, 2001.

COELLI T. J., RAO, P..Total Factor Productivity Growth in Agriculture: A Malmquist Index Analysis of 93 Countries, 1980-2000, Working Paper Series, Centre of Efficiency and Productivity Analysis, 2003.

GOLANY, B., ROLL, Y. An application Procedure for DEA. Omega: The International Journal of Management Science 17, pp. 237-250, 1989. 
KASSAI, S. Utilização da Análise por Envoltória de Dados (DEA) na Análise de Demonstrações Contábeis. Tese (Doutorado) - Faculdade de Economia, Administração e Contabilidade, Universidade de São Paulo, São Paulo, 2002.

KIRCHNER, Leopoldo Heitor Capelini. Avaliação da eficiência dos terminais de contêineres através da análise envoltória de dados e do índice de Malmquist. 2013. 90 f., il. Dissertação (Mestrado Profissional em Regulação e Gestão de Negócios)—Universidade de Brasília, Brasília, 2013.

SOLLERO, M. K. V. Avaliação de Empresas de Distribuição de Energia Elétrica através da Análise Envoltória de Dados com Integração das perspectivas do regulamento e das Concessionárias. COPPE/UFRJ, Rio de Janeiro, RJ, Brasil, 2007.

BANA E COSTA, C. A.; ENSSLIN, L.; CORRÊA, E. C.; MONTIBELLER NETO, G. e ZANELLA, I. J. 1996 a. Construção de um Modelo Multicritérios de Apoio à Decisão Utilizando a Metodologia MACBETH - Estudo de Caso, in SOBRAPO, Rio de Janeiro, Anais, p.248-253.

BANA E COSTA, C. A.; ENSSLIN, L.; COSTA, A. P.; MARTINS, F. M.; HOLZ, E.; SILVA JR., F. F. 1996b. Seleção de Variedades de Arroz para Semeadura - Uma aplicação MCDA - MACBETH, in SOBRAPO, Rio de Janeiro, Anais, p.254-259.

DETONI, M. M. M. L. 1996. Aplicação de Metodologia Multicritério de Apoio à Decisão na Definição de Características de Projetos de Construção. Florianópolis: Dissertação submetida à Universidade Federal de Santa Catarina para a obtenção do Grau de Mestre em Engenharia, Programa de Pós-Graduação em Engenharia de Produção.

GOMES, L. F. A. M., ARAYA, M. C. G., CARIGNANO, C. 2004. Tomada de Decisões em Cenários Complexos, Ed. Pioneira Thompson Learning, São Paulo.

GOMES, L. F. A. M, GOMES, C. F. S., ALMEIDA, A. T. 2006. Tomada de Decisão Gerencial: Enfoque Multicritério, Ed. Atlas, São Paulo, pp.289.

LOBO, MSC; SILVA, ACM; FISZMAN, R; LINS, M. P. E. .Impacto da reforma de financiamento de hospitais de ensino no Brasil. Revista de Saúde Pública / Journal of Public Health, v. 43, p. 437-445, 2009.

MOREIRA, M.A. 2006. A teoria da aprendizagem significativa e suas implementações em sala de aula. Brasília: Editora Universidade de Brasília.
NOVAK, J.D. 1998 "Learning, Creating, and using Knowledge: Concept Maps as Facilitative Tools in Schools and Corporations", Lawrence Erbaum Associates, NJ.

OKADA, A., BUCKINGHAM SHUM, S. AND SHERBORNE, T. 2008. "Knowledge Cartography: Software Tools and Mapping Techniques". (Eds.). Springer: Advanced Information and Knowledge Processing Series. pp. 25-46

RIBEIRO, J. R. ET AL. 2007. “Applications of classical AHP and DEA method select suppliers in capital goods". In: $9^{\text {th }}$ International Symposium on the Analytic Hierarchy Process, Viña Del Mar, Chile. Proceedings of 9th ISAHP, 2007, pp. 10

ROSENHEAD, J.; MINGERS, J. 2001. "Rational analysis for a problematic world: problem-structuring methods for complexity, uncertainty and conflict". 375p. 2. Ed. West Sussex: John Willey \& Sons.

ROY, B., BOUYSSOU, D. 1993. Aide Multicritère à la Décision: Méthodes et Cas, Economica, Paris.

ROY, BERNALD; VANDERPOOTEN, DANIEL. The European school of MDCA: Emergence, basic features and current works. Paris: Université Paris Dauphine. 2005.

RUIZ-MORENO, L.; SONZOGNO, M.C.; BATISTA, S.H.S. E BATISTA, N.A. 2007. Mapa conceitual: ensaiando critérios de análise. Ciência Educação, 13, 453-463.

SAATY, T. L. AND OZDEMIR, M. S. 2003, "Why the Magic Number Seven Plus or Minus Two," Mathematical and Computer Modeling, 38, pp. 233-244.

SAATY, T. L. 1990. "How to Make a Decision: The Analytic Hierarchy Process". European Journal of Operational Research, V. 48, pp. 9-26

VEKIRI, I. 2002. "What Is the Value of Graphical Displays in Learning?" Ed.. Psychol. Rev., 14, 261.

WU, A., CAO, Y., \& LIU, B. Energy efficiency evaluation for regions in China: an application of DEA and Malmquist indices. Energy Efficiency. in print. 2013. 